\title{
Rationalizing Denominators Using Gröbner Bases
}

\author{
Dongmei Li $\mathbb{D}$, Man Wu, Jinwang Liu, and Yiman Gao \\ School of Mathematics and Computing Sciences, Hunan University of Science and Technology, Xiangtan 411201, Hunan, China \\ Correspondence should be addressed to Dongmei Li; dmli@hnust.edu.cn
}

Received 29 May 2021; Revised 22 November 2021; Accepted 29 November 2021; Published 6 January 2022

Academic Editor: Zhiwei Gao

Copyright $\odot 2022$ Dongmei Li et al. This is an open access article distributed under the Creative Commons Attribution License, which permits unrestricted use, distribution, and reproduction in any medium, provided the original work is properly cited.

The problem of rationalizing denominators for two types of fractions is discussed in the paper. By using the theory and algorithms of Gröbner bases, we first introduce a method to rationalize the denominators of fractions with square root and cube root, and then, for the denominators with higher radical of the general form, the problem of rationalizing denominators is converted into the related problem of finding the minimal polynomials. Some interesting results and an executable algorithm for rationalizing the denominator of these type fractions are presented. Furthermore, an example is also established to illustrate the effectiveness of the algorithm.

\section{Introduction}

A typical topic in algebra is rationalizing denominators [1]. Rationalizing the denominator avoids the problem known as "subtrative cancellation," deals with the problem of recognizing equivalent expressions, and is commonly used in many of computer algebra systems. Also, rationalizing expression has applications in calculus [2]. More importantly, by rationalizing the denominator, mathematical operations and practical problems can be approximated more accurately [3]. Thus, there are circumstances in which it is advantageous to rationalize an expression.

The general method of denominator rationalization is to seek the rationalized factor of the denominator first and then multiply the numerator and denominator by this factor at the same time [4]. Using this method, the denominator rationalization of quadratic radical fraction has been solved. However, it is difficult to find the rationalized factor for an expression which contains a root higher than a square root [5]. Therefore, a lot of literatures tend to deal with some special case of this problem. In 1929, Paradiso [6] showed that theoretically, in all cases, and practically, in many cases, a rationalizing factor may be found by the method of undetermined coefficients. In 1970, Fateman presented an algorithm named RADCAN that is implemented in MACSYMA for the simplification of expressions containing radicals [7]. Zhou rationalized the denominator for a class of fractions $1 / g(u)$ by theory of minimal polynomials in 1986, where $g(u)$ is a polynomial whose coefficient are rational and $u$ is a complex root of a nonzero rational polynomial [8]. In 1989, Ma showed the possibility of denominator's rationalization of the irrational expressions: $A$ / $\left(a_{1} \sqrt[n 1]{b_{1}}+a_{2} \sqrt[n 2]{b_{2}}+\cdots+a_{m} \sqrt[n m]{b_{m}}+c\right)$, where $A$ is the combination of radical and rational addition, subtraction, and multiplication and $a_{1} \sqrt[n 1]{b_{1}}+a_{2} \sqrt[n 2]{b_{2}}+\cdots+a_{m} \sqrt[n m]{b_{m}}+c$ is an algebraic element in the field of rational numbers [9]. In 2000, Liu rationalized the denominator for a class of algebraic fractions as follows $1 / f(\sqrt[n+1]{c})=1 /\left(a_{0}+a_{1} \sqrt[n+1]{c}+\cdots+\right.$ $a_{n} \sqrt[n+1]{c^{n}}$ ) (where $f(x)=a_{0}+a_{1} x+\cdots+a_{n} x^{n}, c, a_{1}, a_{2}$, $\left.\ldots, a_{n} \in Q, c>0\right)$ using the knowledge of determinants [10]. In 2002, using polynomials, Tang also discussed the algebraic fractions whose denominator is the same with the above fraction [11]. And in 2015, Berele and Catoiu produced an exact formula for rationalizing any fraction whose denominator is a linear combination with rational coefficients of square roots of rational numbers [1].

Existing results mentioned above mainly deal with several kinds of denominators rationalization of radical fractions by using related theory of polynomials. And many other kinds of denominators of irrational fraction are unsolved, such as $1 /(x+5 \sqrt[7]{8}+6 \sqrt[4]{9})$. This kind of fraction is a very common form in mathematical calculation, so it is of 
high application value to study a general method to rationalize the denominator. In this paper, we will investigate this by the theory of Gröbner bases as it is an important tool to solve many problems in polynomial ideal $[12,13]$. And it has been implemented in many computational softwares including Singular, Maple, CoCoA, Mathematica, Macaulay 2, etc. Many fundamental problems in commutative algebra, computational algebraic number theory, algebraic geometry, graph theory, image processing, cryptography and encoding, and science and engineering can be solved by it algorithmically [13-23]. And the minimal polynomial can be obtained by the reduced Gröbner basis algorithm easily. Based on this and results mentioned above, we consider using the theory of Gröbner bases to explore the relationship between minimal polynomials and denominator rationalization and discuss mainly denominator rationalization of the fraction with the form as

$$
\frac{1}{x+c_{1} \cdot \sqrt[m]{a}+c_{2} \cdot \sqrt[n]{b}}
$$

where $m, n \in N^{+}, m, n \geq 2, a, b, c_{1}, c_{2} \in Q, a, b>0$. We hope to establish a simplified method for rationalizing the denominator of this type fraction.

The rest of the paper is organized as follows. We present some preliminary knowledge, basic concepts, and a special method to rationalize the denominators with square root and cube root in Section 2. Main results on rationalizing the denominator of a type fraction are shown in Section 3. A simplified algorithm and an example established to illustrate the algorithm are given in Section 4 . Section 5 concludes the paper.

\section{Denominators with Square Root and Cube Root}

In what follows, $K\left[x_{1}, x_{2}, \ldots, x_{n}\right]$ will denote the polynomial ring in $n$ variables $x_{1}, x_{2}, \ldots, x_{n}$ with coefficients in a field $K, L / K$ will be an algebraic extension of the field $K$, and $[L: K]$ will denote the extension times of the extension field $L / K$. $Q$ will denote the rational number field, $N$ will be the set of integers, and $\operatorname{lcm}(m, n)$ will denote the least common multiple of $m$ and $n$. For a nonzero polynomial $f(x) \in K\left[x_{1}, x_{2}, \ldots, x_{n}\right]$, we use $l t(f), l c(f)$, and $l p(f)$ to denote the leading term, the leading coefficient, and the leading monomial of $f(x)$, respectively. For a set $F \subset K\left[x_{1}, x_{2}, \ldots, x_{n}\right]$, we denote $l t(F)=\{l t(f) \mid f \in F\}$. Then, we introduce several related definitions and algorithm.

Definition 1. Let $I$ be an ideal in $K\left[x_{1}, x_{2}, \ldots, x_{n}\right]$. A finite subset $G$ of $I$ is called a Gröbner basis of $I$ if

$$
\langle l t(G)\rangle=\langle l t(I)\rangle .
$$

Definition 2 (see [13]). Let $f, g \in K\left[x_{1}, x_{2}, \ldots, x_{n}\right], f, g \neq$ 0 , and $L=\operatorname{lcm}(\operatorname{lp}(f), \operatorname{lp}(g))$, and the S-polynomial of $f$ and $g$ is defined as (Algorithm 1)

$$
S(f, g)=\frac{L}{l t(f)} f-\frac{L}{l t(g)} g .
$$

This section focuses on the problem of rationalizing the denominator with square root and cube root. For the sake of convenience in researching the problem, we put the coefficient in the radical sign, and then, the fraction can be reduced to one of the following forms:

$$
\begin{aligned}
& \text { (1) } \frac{1}{x+\sqrt[2]{a}+\sqrt[3]{b}}(a>0 \text {, and } a, b \in Q), \\
& \text { (2) } \frac{1}{x-\sqrt[2]{a}+\sqrt[3]{b}}(a>0 \text {, and } a, b \in Q) .
\end{aligned}
$$

We first consider the denominators rationalization of form (1).

Lemma 1 (see [13]). Let $G=\left\{g_{1}, g_{2}, \ldots, g_{s}\right\}$ be a subset of an ideal $I \in K\left[x_{1}, x_{2}, \ldots, x_{n}\right]$, and then, $G$ is a Gröbner bases for $I$ if and only if $S\left(g_{i}, g_{j}\right) \stackrel{G}{\longrightarrow} 0$, for all $i \neq j, 1 \leq i, j \leq s$.

Theorem 1. Let I be an ideal in $K\left[x_{1}, x_{2}, \ldots, x_{n}\right]$, and then, $f g-1 \in I \Longleftrightarrow 1 \in\langle I, f\rangle$.

Proof. If $f g-1 \in I$, then there exists $h \in I$, such that $f g-1=h$, that is, $1=f g-h \in\langle I, f\rangle$.

In turn, if $1 \in\langle I, f\rangle$, then there exists $g \in K\left[x_{1}, x_{2}, \ldots, x_{n}\right]$ and $h \in I$, such that $1=f g+h$, that is, $f g-1=-h \in I$.

Next, we introduce how to rationalize the denominator of fraction as form (1). First, consider the following ideal:

$$
I=\left\langle y_{1}^{2}-a, y_{2}^{3}-b\right\rangle \subseteq Q(x)\left[y_{1}, y_{2}\right],
$$

where $Q(x)\left[y_{1}, y_{2}\right]$ denotes the polynomial ring in variables $y_{1}$ and $y_{2}$ on $Q(x)$ and $Q(x)$ is an extension of the field $Q$ with $x$ as a variable. Let

$$
f_{1}\left(y_{1}, y_{2}\right)=y_{1}^{2}-a, f_{2}\left(y_{1}, y_{2}\right)=y_{2}^{3}-b \text {. }
$$

In the following, multivariate polynomials $h(x, y)$ are denoted as $h$ for convenience. And the term order of the multivariate polynomial ring is the lexicographical ordering, which is defined by $y_{1}>y_{2}$.

Note that

$$
S\left(f_{1}, f_{2}\right)=b y_{1}^{2}-a y_{2}^{3} \stackrel{f_{2}}{\longrightarrow} b y_{1}^{2}+a b \stackrel{f_{1}}{\longrightarrow} 0 .
$$

By Lemma 1, we have that $\left\{f_{1}, f_{2}\right\}$ is a Gröbner basis of I. Let 


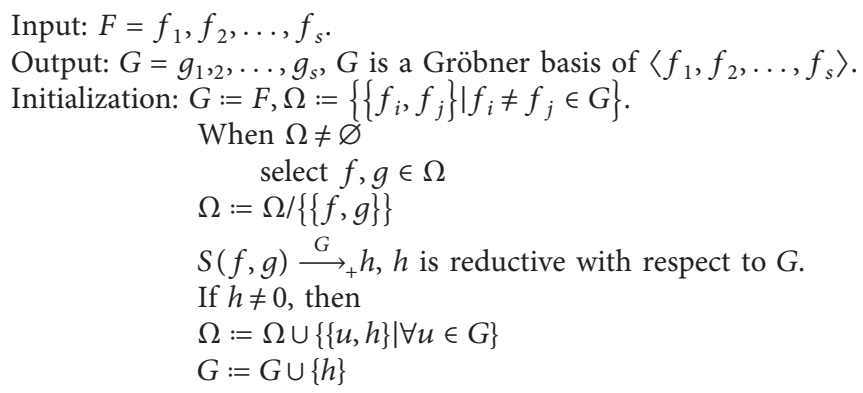

Algorithm 1: Buchberger algorithm [14].

$$
f_{3}\left(y_{1}, y_{2}\right)=x+y_{1}+y_{2},
$$

$y_{1}=\sqrt{a}, y_{2}=\sqrt[3]{b}$, and then, $f_{3}(\sqrt{a}, \sqrt[3]{b})=x+\sqrt{a}+\sqrt[3]{b}$, which is the denominator we want to deal with. Replace $f$ by $f_{3}$ in Theorem 1 , and then, the condition $f_{3} \cdot g-1 \in I$ is equivalent to $f_{3}(\sqrt{a}, \sqrt[3]{b}) \cdot g(\sqrt{a}, \sqrt[3]{b})=1$, where $g(\sqrt{a}, \sqrt[3]{b}) \in Q[\sqrt{a}, \sqrt[3]{b}]$ is the polynomial we need.

So, the key issue is whether $1 \in\left\langle I, f_{3}\right\rangle$, that is, whether $1 \in\left\langle f_{1}, f_{2}, f_{3}\right\rangle$. If true, we can rationalize the denominator of $1 /(x+\sqrt[2]{a}+\sqrt[3]{b})$ by using this method.

Now, we use Buchberger algorithm to obtain the Gröbner basis of $J=\left\langle f_{1}, f_{2}, f_{3}\right\rangle$ and then determine whether $1 \in\left\langle f_{1}, f_{2}, f_{3}\right\rangle$.

Initialize

$f_{1}=y_{1}^{2}-a, f_{2}=y_{2}^{3}-b$, and $f_{3}=x+y_{1}+y_{2}$, and using the algorithm, we get the Gröbner basis of $J$ is

$$
\left\{f_{1}, f_{2}, f_{3}, f_{4}, f_{5}, f_{6}\right\} \text {, }
$$

where

$$
\begin{aligned}
& f_{4}=y_{2}^{2}-x y_{1}+x y_{2}-a, \\
& f_{5}=\left(a+3 x^{2}\right) y_{2}+2 x^{3}-2 a x-b, \\
& f_{6}=\frac{-\left(2 x^{3}-2 a x-b\right)^{3}}{\left(3 x^{2}+a\right)^{3}}-b .
\end{aligned}
$$

Observe $f_{6}$, and it is easy to find that $f_{6}$ is independent of $y_{1}$ and $y_{2}$, and thus, $f_{6} \in Q(x)$. Note that $Q(x)$ is a field, and thus, $f_{6}^{-1}=1 / f_{6} \in Q(x)$. Consequently, $1=f_{6} \cdot 1 /$ $f_{6} \in J$. Therefore, we can calculate the rationalizing result of $1 /(x+\sqrt{a}+\sqrt[3]{b})$.

Then, express $f_{6}$ as the combination of $f_{1}, f_{2}, f_{3}, f_{4}, f_{5}$ :

$$
f_{6}=f_{2}-\left(\frac{y_{2}^{2}}{3 x^{2}+a}+\frac{-2 x^{3}+2 a x+b}{\left(3 x^{2}+a\right)^{2}}+\frac{\left(2 x^{3}-2 a x-b\right)^{2}}{\left(3 x^{2}+a\right)^{3}}\right) \cdot f_{5}
$$

that is,

$$
\frac{f_{2}-\left(y_{2}^{2} /\left(3 x^{2}+a\right)+\left(-2 x^{3}+2 a x+b\right) /\left(3 x^{2}+a\right)^{2}+\left(2 x^{3}-2 a x-b\right)^{2} /\left(3 x^{2}+a\right)^{3}\right) \cdot f_{5}}{f_{6}}=1 .
$$

Hence, divide both sides of equation (13) by $x+\sqrt{a}+\sqrt[3]{b}$, and substitute the value of $f_{2}, f_{5}$ and $y_{1}=\sqrt{a}, y_{2}=\sqrt[3]{b}$ into the equation, and then, we obtain 


$$
\begin{aligned}
\frac{1}{x+\sqrt{a}+\sqrt[3]{b}=} & \frac{\left(2 x^{3}-2 a x-b\right)^{2} \cdot 2 x^{2}+b x \cdot\left(3 x^{2}+a\right)^{2}+b\left(2 x^{3}+2 a x+b\right) \cdot\left(3 x^{2}+a\right)}{\left(2 x^{3}-2 a x-b\right)^{3}+b\left(3 x^{2}+a\right)^{3}} \\
& +\frac{b\left(3 x^{2}+a\right)^{2}-2 x \cdot\left(2 x^{3}-2 a x-b\right)^{2}}{\left(2 x^{3}-2 a x-b\right)^{3}+b\left(3 x^{2}+a\right)^{3}} \cdot \sqrt{a} \\
& +\frac{-b\left(3 x^{2}+a\right)^{2}+2 x^{2} \cdot\left(-2 x^{3}+2 a x+b\right) \cdot\left(3 x^{2}+a\right)+x\left(2 x^{3}-2 a x-b\right)^{2}}{\left(2 x^{3}-2 a x-b\right)^{3}+b\left(3 x^{2}+a\right)^{3}} \cdot \sqrt[3]{b} \\
& +\frac{2 x^{2} \cdot\left(3 x^{2}+a\right)^{2}+x \cdot\left(3 x^{2}+a\right) \cdot\left(-2 x^{3}+2 a x+b\right)-\left(2 x^{3}-2 a x-b\right)^{2}}{\left(2 x^{3}-\sqrt[3]{b^{2}}\right.} \\
& +\frac{2 x \cdot\left(2 x^{3}-2 a x-b\right) \cdot\left(3 x^{2}+a\right)+\left(2 x^{3}-2 a x-b\right)^{3}}{\left(2 x^{3}-2 a x-b\right)^{3}+b\left(3 x^{2}+a\right)^{3}} \cdot \sqrt{a} \cdot \sqrt[3]{b} \\
& +\frac{-2 x \cdot\left(3 x^{2}+a\right)^{2}+\left(-2 x^{3}+2 a x+b\right) \cdot\left(3 x^{2}+a\right)}{\left(3 x^{2}+a\right)^{3}} \cdot \sqrt{a} \cdot \sqrt[3]{b^{2}} \cdot
\end{aligned}
$$

Using Matlab to simplify the equation above, we obtain

$$
\begin{aligned}
\frac{1}{x+\sqrt{a}+\sqrt[3]{b}=} & \frac{1}{x^{6}-3 a x^{4}+2 b x^{3}+3 a^{2} x^{2}+6 a b x+b^{2}-a^{3}}\left(x^{5}-2 a x^{3}+b x^{2}\right. \\
& +a^{2} x+a b+\left(-x^{4}+2 a x^{2}+2 b x-a^{2}\right) \cdot \sqrt{a}+\left(-x^{4}-b x+a^{2}\right) \\
& \cdot \sqrt[3]{b}+\left(x^{3}+3 a x+b\right) \cdot \sqrt[3]{b^{2}}+\left(2 x^{3}-2 a x-b\right) \cdot \sqrt{a} \cdot \sqrt[3]{b} \\
& \left.+\left(-3 x^{2}-a\right) \cdot \sqrt{a} \cdot \sqrt[3]{b^{2}}\right)
\end{aligned}
$$

For form (2), we just need to change $f_{3}\left(y_{1}, y_{2}\right)=x+$ $y_{1}+y_{2}$ to $f_{3}\left(y_{1}, y_{2}\right)=x-y_{1}+y_{2}$, and the other steps are the same.

\section{Denominators with Higher Radical}

In this section, we focus on rationalizing the denominator of general form as

$$
\frac{1}{x+c_{1} \cdot \sqrt[m]{a}+c_{2} \cdot \sqrt[n]{b}}
$$

where $m, n \in N^{+}, m, n \geq 2, a, b, c_{1}, c_{2} \in Q, a, b>0$.

By the method in Section 2, we first construct the ideal $J=\left\langle f_{1}, f_{2}, f_{3}\right\rangle \in Q(x)\left[y_{1}, y_{2}\right]$, where $f_{1}=y_{1}^{m}-a$, $f_{2}=y_{2}^{n}-n$, and $f_{3}=x+c_{1} y_{1}+c_{2} y_{2}$. Then, compute the Gröbner basis $G$ and the reduced Gröbner basis $G_{0}$ of $J$. If $G_{0}=\{1\}$, then calculate $f_{s}=G \cap Q(x)$. Finally, express $f_{s}$ as a combination of $G /\left\{f_{s}\right\}$.

However, there are two important uncertainties, one is whether $G_{0}=\{1\}$ and the other is whether $f_{s}$ is a combination of $G /\left\{f_{s}\right\}$. So, this method can only solve the case when $c_{1}, c_{2}, m, n$ are specific values. Because of this limitation, we hope to find another method for the general form.

First, we introduce several related definitions and lemmas.

Definition 3 (see [24]). Let $L / K$ be an algebraic extension of the field $K,[L: K]=n$. Suppose $\sigma_{i}: L \longrightarrow C(1 \leq i \leq n)$ is $n$ $\mathrm{K}$-insertion of $L$, where $C$ is the complex field. For $\alpha \in L$, define

$$
T_{L / K}(\alpha)=\sum_{i=1}^{n} \sigma_{i}(\alpha),
$$

as the trace of $\alpha$ to the expansion $L / K$.

Definition 4 (see [24]). Let $L / K$ be an algebraic extension of the field $K$. Suppose $\sigma_{1}, \ldots, \sigma_{n}$ is the K-insertion of $L$ and $\alpha_{1}, \ldots, \alpha_{n} \in L$, and we define

$$
d_{L / K}\left(\alpha_{1}, \ldots, \alpha_{n}\right)=\operatorname{det}^{2}\left(\sigma_{i}\left(\alpha_{j}\right)\right), 1 \leq i \leq n, 1 \leq j \leq n,
$$

as the discriminant of $\left\{\alpha_{1}, \ldots, \alpha_{n}\right\}$ for the expansion $L / K$. 
Definition 5. Let $K$ be a field, $u \in K$, and $f(x) \in K[x]$ be a polynomial. We call $f(x)$ is the minimal polynomial of $u$ in $K$ if $f(x)$ satisfies the following:
(1) $f(x)$ is monic and $f(u)=0$
(2) If $g(x) \in K[x]$ and $g(u)=0$, then $f(x) \mid g(x)$

Lemma 2 (see [24]). Let $L / K$ be the expansion of number field and $[L: K]=n$. Suppose $\alpha \in L, f(x)=x^{m}-c_{1} x^{m-1}+$ $\cdots+(-1)^{m} c_{m}$ is the minimal polynomial of $\alpha$ in $K$, where $m=[K(\alpha): K]$, and then, $T_{L / K}(\alpha)=n c_{1} / m$.

Lemma 3 (see [23]). Let $d_{L / K}\left(\alpha_{1}, \ldots, \alpha_{n}\right)$ be the discriminant of element $\left\{\alpha_{1}, \ldots, \alpha_{n}\right\}$ for the expansion $L / K$, and then, $d_{L / K}\left(\alpha_{1}, \ldots, \alpha_{n}\right) \neq 0$ if and only if $\alpha_{1}, \ldots, \alpha_{n}$ is K-linearly independent.

Lemma 4 (see [25], Eisenstein criterion). Let $f(x)=a_{n} x^{n}+$ $a_{n-1} x^{n-1}+\cdots+a_{1} x+a_{0}$ be an integral coefficient univariate polynomial. If there is a prime number $p$ such that

(1) $p+a_{n}$

(2) $p \mid a_{n-1}, a_{n-2}, \ldots, a_{0}$

(3) $p+a_{0}^{2}$ Q.

Then, $f(x)$ is irreducible over the rational number field

Using the lemmas above, we can prove the following results.

Theorem 2. If $p$ is prime number, then $f(x)=x^{m}-p$ is the minimal polynomial for $\sqrt[m]{p}$ in rational number filed $Q$, where $m \in N^{+}$and $m \geq 2$.

Proof. It is straightforward that $\sqrt[m]{p}$ is a root of $f(x)=x^{m}-p=0$. In the following, we prove that $f(x)$ is irreducible.

Let $g(x)=f(x+p)=x^{m}+m p x^{n-1}+\cdots+C_{m}^{i} p^{i} x^{m-i}+$ $\cdots+p^{m}-p$, where $C_{m}^{i}=m ! /(i !(m-i) !)$. It is obvious that $p \nmid 1, p \mid C_{m}^{i} p^{i}(1 \leq i \leq m-1)$, and $p \mid\left(p^{m}-p\right)$. In fact, we can prove that $p^{2} \downarrow\left(p^{m}-p\right)$. Suppose $p^{2} \mid\left(p^{m}-p\right)$, and then, $p \mid\left(p^{m-1}-1\right)$. Combining $p \mid p^{m-1}\left(m \in N^{+}, m \geq 2\right)$, we have that $p \mid\left(p^{m-1}-\left(p^{m-1}-1\right)\right)$, that is, $p \mid 1$, and this is a contradiction. So, $p^{2}+\left(p^{m}-p\right)$. By the Eisenstein criterion, $g(x)$ is irreducible, so $f(x)$ is irreducible, and it is the minimal polynomial for $\sqrt[m]{p}$ in $Q$.

Theorem 3. If $p_{1}, p_{2}, \ldots, p_{s}$ are different prime numbers, then

$$
\sqrt[m 1]{p_{1}^{t_{1}}} \cdot \sqrt[m 2]{p_{2}^{t_{2}}}, \cdots \sqrt[m s]{p_{s}^{t_{s}}},\left(t_{i}<m_{i}, t_{i}, m_{i} \in N^{+}, i=1,2, \ldots, s\right),
$$

is an irrational number.

Proof. It is straightforward that $\sqrt[m 1]{p_{1}^{t_{1}}} \cdot \sqrt[m 2]{p_{2}^{t_{2}}} \cdots \sqrt[m s]{p_{s}^{t_{s}}}$ is a real number. In the following, we prove that it is an irrational number. Suppose it is a rational number, and set $f(x)=x^{m_{1} m_{2} \cdots m_{s}}-p_{1}^{t_{1} m_{2} \cdots m_{s}} p_{2}^{m_{1} t_{2} \cdots m_{s}} \cdots p_{s}^{m_{1} m_{2} \cdots t_{s}} . \quad$ Then, $\sqrt[m 1]{p_{1}^{t_{1}}} \cdot \sqrt[m 2]{p_{2}^{t_{2}}} \cdots \sqrt[m s]{p_{s}^{t_{s}}}$ is a positive rational root of $f(x)=0$ Note that $f(x)$ is an integral coefficient polynomial and lc $(f)=1$, and then, the positive rational root of $f(x)$ must be a factor of its constant term. So, it has the form as $p_{1}^{n_{1}} p_{2}^{n_{2}} \cdots p_{s}^{n_{s}}$, where $0 \leq n_{i} \leq m_{1} \cdots m_{i-1} t_{i} m_{i+1} \cdots m_{s}, n_{i} \in N$, $i=1,2, \ldots, s$. Then, we have that

$$
\sqrt[m 1]{p_{1}^{t_{1}}} \cdot \sqrt[m 2]{p_{2}^{t_{2}}} \cdots \sqrt[m s]{p_{s}^{t_{s}}}=p_{1}^{n_{1}} p_{2}^{n_{2}} \cdots p_{s}^{n_{s}}
$$

From the equation above, we see that some of $n_{i}$ must be 0 . Without loss of generality, we denote the elements whose power exponents are nonzero as $p_{1}, p_{2}, \ldots, p_{q}$, and equation (20) turns into the following:

$$
\sqrt[m 1]{p_{1}^{t_{1}}} \cdot \sqrt[m 2]{p_{2}^{t_{2}}} \cdots \sqrt[m s]{p_{s}^{t_{s}}}=p_{1}^{n_{1}} p_{2}^{n_{2}} \cdots p_{q}^{n_{q}}
$$

that is,

$$
p_{1}^{n_{1}-t_{1} / m_{1}} p_{2}^{n_{2}-t_{2} / m_{2}} \cdots p_{q}^{n_{q}-t_{q} / m_{q}}=p_{q+1}^{t_{q+1} / m_{q+1}} p_{q+2}^{t_{q+2} / m_{q+2}} \cdots p_{s}^{t_{s} / m_{s}} .
$$

Multiply both sides of equation (22) by the $m_{1} m_{2} \cdots m_{s}$ power, and then,

$$
p_{1}^{l_{1}} p_{2}^{l_{2}} \cdots p_{s}^{l_{s}}=p_{q+1}^{l_{q+1}} p_{q+2}^{l_{q+2}} \cdots p_{s}^{l_{s}}
$$

where $l_{i} \in N^{+}, i=1,2, \ldots, s$. Hence, $p_{1} \mid p_{q+1}^{l_{q+1}} p_{q+2}^{l_{q+2}} \cdots p_{s}^{l_{s}}$, and this contradicts $p_{1}, p_{2}, \ldots, p_{s}$ are different prime numbers. So, the conclusion is correct.

Theorem 4. If $p_{1}, \ldots, p_{s}$ are different prime numbers and $m_{1}, \ldots, m_{s}$ are positive integers and no less than 2 , then the finite extension times of $Q\left(\sqrt[m 1]{p_{1}}, \ldots, \sqrt[m s]{P_{s}}\right) / Q$ is no more than $m_{1} m_{2} \ldots m_{s}$.

Proof. Let $F_{i}=Q\left(\sqrt[m 1]{p_{1}}, \sqrt[m_{2}]{p_{2}}, \ldots, \sqrt[m i]{p_{i}}\right), \quad 1 \leq i \leq s$ and $i \in N^{+}$, and then, $F_{i+1}=F_{i}\left(\sqrt[m i+1]{p_{i+1}}\right)$, that is, $F_{i+1}$ can be viewed as the single extension of $F_{i}$. So, $\left[F_{i+1}: F_{i}\right]=\partial\left(g_{i}\right)$, where $\partial\left(g_{i}\right)$ denotes the degree of the polynomial $g_{i}(x)$, and $g_{i}(x)$ is the minimal polynomial of $\sqrt[m i]{p_{i}}$ in the field $F_{i}$. Obviously, $\sqrt[m i]{p_{i}}$ is a root for $f_{i}(x)=x^{m_{i}}-p_{i}, 1 \leq i \leq s$. By the definition of minimal polynomial, we see that $\partial\left(g_{i}\right) \leq \partial\left(f_{i}\right), 2 \leq i \leq s, i \in N^{+}$. From Theorem 3, we see that $f_{1}(x)$ is an irreducible polynomial in $Q$. By the property of domain extension, we have $\left[F_{1}: Q\right]=m_{1}$. Hence, 


$$
\begin{aligned}
{\left[Q\left(\sqrt[m 1]{p_{1}}, \sqrt[m 2]{p_{2}}, \ldots, \sqrt[m s]{p_{s}}\right): Q\right] } & =\left[F_{s}: F_{s-1}\right]\left[F_{s-1}: F_{s-2}\right] \cdots\left[F_{2}: F_{1}\right]\left[F_{1}: Q\right] \\
& =\partial\left(g_{s}\right) \cdot \partial\left(g_{s-1}\right) \cdots \partial\left(g_{2}\right) \cdot m_{1} \\
& \leq m_{1} m_{2} \cdots m_{s}
\end{aligned}
$$

Theorem 5. If $p_{1}, p_{2}, \ldots, p_{s}$ are different prime numbers and $m_{1}, m_{2}, \ldots, m_{s}$ are positive integers and no less than 2, then $x^{m_{s}}-p_{s}$ is the minimal polynomial of $\sqrt[m s]{p_{s}}$ in the field $Q\left(\sqrt[m 1]{p_{1}}, \sqrt[m 2]{p_{2}}, \ldots, \sqrt[m s-1]{p_{s-1}}\right)$.

Proof. Let $b_{i}=\sqrt[m i]{p_{i}}, 1 \leq i \leq s, M=Q\left(\sqrt[m 1]{p_{1}}, \sqrt[m 2]{p_{2}}, \ldots, \sqrt[m s]{p_{s}}\right)$, and in the following, we prove that all the $m_{1} \cdot m_{2} \cdots m_{s}$ elements in set

$$
\begin{aligned}
A & =\left\{b_{1}^{n_{1}} b_{2}^{n_{2}} \cdots b_{s}^{n_{s}} \mid 0 \leq n_{i}\right. \\
& \left.\leq m_{i}-1, n_{i} \in N, i=1,2, \ldots, s\right\} \subseteq M,
\end{aligned}
$$

are linearly independent. First, sort the elements in $A$. It is easy to observe that each element in $A$ corresponds to such an array $\left(n_{1}, n_{2}, \ldots, n_{s}\right)$. So, we can turn the problem into sorting the exponents $\left(n_{1}, n_{2}, \ldots, n_{s}\right)$, where the term order is lexicographic order. We denote the element that corresponds to the largest exponential as $c_{1}$, the element that corresponds to the second largest exponential as $c_{2}, \ldots$, and the element that corresponds to the smallest exponential as $c_{m_{1} m_{2} \cdots m_{s}}$, and then, we have sorted out all the elements in $A$.

Now, we calculate det $\left(T_{M / Q}\left(c_{i} c_{j}\right)\right)$. Suppose $c_{i}=b_{1}^{n_{i_{1}}} b_{2}^{n_{i_{2}}} \cdots b_{s}^{n_{i_{s}}}$ and $c_{j}=b_{1}^{n_{j_{1}}} b_{2}^{n_{j_{2}}} \cdots b_{s}^{n_{j_{s}}}$. If one of the following two situations is true,

(1) $\forall k=1,2, \ldots, s, n_{i_{k}}+n_{j_{k}}=m_{k}$

(2) $\forall k=1,2, \ldots, s, n_{i_{k}}=n_{j_{k}}=0$

We obtain that $c_{i} c_{j} \in Q$ by Theorem 3. Hence, $T_{M / Q}\left(c_{i} c_{j}\right)=n c_{i} c_{j} \neq 0$, where $n=[M: Q]$. Otherwise, there exists $k \in\{1,2, \ldots, s\}$ does not satisfy either of the above two cases, and we can calculate $T_{M / Q}\left(c_{i} c_{j}\right)$ by Lemma 2 . We first want to obtain the minimal polynomial of $c_{i} c_{j}$ in rational number field $Q$. Set

$$
l=\operatorname{lcm}\left(\frac{m_{1}}{\operatorname{gcd}\left(m_{1}, n_{i_{1}}+n_{j_{1}}\right)}, \frac{m_{2}}{\operatorname{gcd}\left(m_{2}, n_{i_{2}}+n_{j_{2}}\right)}, \ldots, \frac{m_{s}}{\operatorname{gcd}\left(m_{s}, n_{i_{s}}+n_{j_{s}}\right)}\right) .
$$

Obviously, $\quad l>1$. Let $q=\left(c_{i} c_{j}\right)^{l} \quad$ and $B=\left\{m \in N^{+} \mid\left(c_{i} c_{j}\right)^{m} \in Q\right\}$, and by Theorem 3, we have that $m_{k} \mid\left(n_{i_{k}}+n_{j_{k}}\right) m$, for any $m \in B$, where $k=1,2, \ldots, s$. Hence, $p$ is the smallest element in $B$.

In the following, we prove that $f(x)=x^{l}-q$ is the minimal polynomial of $c_{i} c_{j}$ in the rational number field $Q$.

It is straightforward that $f\left(c_{i} c_{j}\right)=\left(c_{i} c_{j}\right)^{l}-q=0$. We factorize $f(x)$ in the complex field $C$ as

$$
f(x)=(x-\sqrt[l]{q})(x-\varepsilon \sqrt[l]{q}) \cdots\left(x-\varepsilon^{l-1} \sqrt[l]{q}\right)
$$

where $\varepsilon=e^{2 \pi i / l}=\cos (2 \pi / n)+i \sin (2 \pi / n)$.

Suppose $f(x)$ is reducible in $Q$, and then, some constant terms of the linear factor in the decomposition above are rational numbers, that is, there is a $t \in N^{+}$and $k \in N$ such that $(-1)^{t} \varepsilon^{k} q^{t / l} \in Q \subseteq R$, i.e., $e^{2 k \pi i / l}=\cos (2 k \pi / l)+i \sin (2 k \pi /$ l) $\in R$. So, $\sin (2 k \pi / l)=0$, that is, $k=\ln / 2, n \in Z$. Hence, $\varepsilon^{k}=\cos (2 k \pi / l)=\cos (n \pi)= \pm 1, n \in Z$, and there is an $r \in Q$ such that $q^{t / l}=r$. Note that $q=\left(c_{i} c_{j}\right)^{l}$, and then, $\left(c_{i} c_{j}\right)^{t}=r \in Q(t<l)$, and this contradicts the selection of $l$. Thus, $f(x)=x^{l}-q$ is irreducible in $Q$, and then, $f(x)$ is the minimal polynomial of $c_{i} c_{j}$ in rational number field $Q$. Combined with Lemma 2, we see that $T_{M / Q}\left(c_{i} c_{j}\right)=0$.

Based on the discussion above, we obtain that $d_{M / Q}\left(c_{1}, c_{2}, \ldots, c_{m_{1} m_{2} \cdots m_{s}}\right)=\operatorname{det}\left(T_{M / Q}\left(c_{i} c_{j}\right)\right) \neq 0$. Then, all elements in set $A$ are linearly independent in $Q$ by Lemma 3. Combined with Theorem 4, we see that

$$
\left[Q\left(\sqrt[m 1]{p_{1}}, \sqrt[m 2]{p_{2}}, \ldots, \sqrt[m s]{p_{s}}\right): Q\right]=m_{1} m_{2} \cdots m_{s}
$$

So, $x^{m_{s}}-p_{s}$ is the minimal polynomial of $\sqrt[m s]{p_{s}}$ in the field $Q\left(\sqrt[m 1]{p_{1}}, \ldots, \sqrt[m s-1]{p_{s-1}}\right)$

Based on the results above, we can present the steps for rationalizing denominators of the following form:

$$
\frac{1}{x+c_{1} \sqrt[m]{a}+c_{2} \sqrt[n]{b}}
$$

where $m$ and $n$ are integers and $a, b \in Q^{+}$and $c_{1}, c_{2} \in Q$.

Step 1: write $a$ and $b$ as fractions in the lowest term, $a=d_{1} / e_{1}$ and $b=d_{2} / e_{2}$. Then, decompose $d_{1}, e_{1}, d_{2}, e_{2}$ into power product of different prime factors, respectively. We use $p_{1}, p_{2}, \ldots, p_{t}$ to denote the common prime factors of $d_{1}$ and $d_{2}, q_{1}, q_{2}, \ldots, q_{k}$ to denote the different prime factors in $d_{1}, e_{1}$, and $v_{1}, v_{2}, \ldots, v_{s}$ to denote the different prime factors in $d_{2}, e_{2}$.

Step 2: let $l=\operatorname{lcm}(m, n)$, and

$$
\begin{aligned}
\alpha_{1} & =\sqrt[l]{p_{1}}, \alpha_{2}=\sqrt[l]{p_{2}}, \ldots, \alpha_{t}=\sqrt[l]{p_{t}}, \\
\alpha_{t+1} & =\sqrt[m]{q_{1}}, \alpha_{t+2}=\sqrt[m]{q_{2}}, \ldots, \alpha_{t+w}=\sqrt[m]{q_{w}} \\
\alpha_{t+w+1} & =\sqrt[n]{r_{1}}, \alpha_{t+w+2}=\sqrt[n]{r_{2}}, \ldots, \alpha_{t+w+s}=\sqrt[n]{r_{s}} .
\end{aligned}
$$

We construct the rational extension field:

$$
M=Q\left(\alpha_{1}, \ldots, \alpha_{t}, \alpha_{t+1}, \ldots, \alpha_{t+w}, \ldots, \alpha_{t+w+s}\right) .
$$


Let $\alpha=c_{1} \sqrt[m]{a}+c_{2} \sqrt[n]{b}$, and there are $f\left(x_{1}, \ldots\right.$, $\left.x_{t+w+s}\right), g\left(x_{1}, \ldots, x_{t+w+s}\right) \in Q\left[x_{1}, \ldots, x_{t+w+s}\right]$ such that

$$
\alpha=f\left(\alpha_{1}, \ldots, \alpha_{t+w+s}\right) / g\left(\alpha_{1}, \ldots, \alpha_{t+w+s}\right) .
$$

According to Theorem 2, we have that $h_{1}=x_{1}^{l}-$ $p_{1} \in Q\left[x_{1}\right]$ is the minimal polynomial of $\alpha_{1}$ in the rational number field $Q$. It is also known, by Theorem $5, h_{i}=x^{l}-$ $p_{i} \in Q\left(\alpha_{1}, \ldots, \alpha_{i-1}\right)\left[x_{i}\right]$ is the minimal polynomial of $\alpha_{i}$ in $Q\left(\alpha_{1}, \ldots, \alpha_{i-1}\right) \quad$ for $\quad 2 \leq i \leq t, \quad h_{i}=x^{m}-q_{i-t} \in Q\left(\alpha_{1}, \ldots\right.$, $\left.\alpha_{i-1}\right)\left[x_{i}\right]$ is the minimal polynomial of $\alpha_{i}$ in $Q\left(\alpha_{1}, \ldots, \alpha_{i-1}\right)$ for $t+1 \leq i \leq t+s$, and $h_{i}=x^{n}-v_{i-t-w} \in Q\left(\alpha_{1}, \ldots, \alpha_{i-1}\right)\left[x_{i}\right]$ is the minimal polynomial of $\alpha_{i}$ in $Q\left(\alpha_{1}, \ldots, \alpha_{i-1}\right)$ for $t+w+1 \leq i \leq t+w+s$.

Next, we construct the following homomorphic maps: $\varphi_{i}: Q\left[x_{1}, x_{2}, \ldots, x_{i}\right] \longrightarrow Q\left(\alpha_{1}, \alpha_{2}, \ldots, \alpha_{i-1}\right)\left[x_{i}\right], 2 \leq i \leq t+w+s$.

For any $f=\sum \lambda_{\beta} x_{1}^{\beta_{1}} x_{2}^{\beta_{2}} \ldots x_{i}^{\beta_{i}} \in Q\left[x_{1}, x_{2}, \ldots, x_{i-1}\right]$, where $\lambda_{\beta} \in Q, \beta=\left(\beta \beta_{1}, \beta_{2}, \ldots, \beta_{i}\right) \in N^{i}$. Define

$$
\varphi_{i}(f)=\sum_{\beta} \lambda_{\beta} \alpha_{1}^{\beta_{1}} \alpha_{2}^{\beta_{2}} \cdots \alpha_{i-1}^{\beta_{i-1}} x_{i}^{\beta_{i}} .
$$

Under this map, the corresponding preimage of $h_{i} \in Q\left(\alpha_{1}, \alpha_{2}, \ldots, \alpha_{i-1}\right)\left[x_{i}\right]$ is itself, where $2 \leq i \leq t+w+s$.

Step 3: we find the minimal polynomial of $\alpha$ in $Q$ according to [26].

The term order $\prec$ in $Q\left[x_{1}, \ldots, x_{t}, x_{t+1}, \ldots, x_{t+w+s}, y\right]$ is the lexicographical ordering defined by $y<x_{t+w+s}<\cdots<x_{t+1}<x_{t}<\cdots<x_{1}$. Under this term order, we calculate the reduced Gröbner basis $G$ of the ideal

$$
I=\left\langle h_{1}, h_{2}, \ldots, h_{t+w+s}, g \cdot y-f\right\rangle,
$$

where

$$
h_{i}= \begin{cases}x^{l}-p_{i}, & 1 \leq i \leq t, \\ x^{m}-q_{i-t}, & t+1 \leq i \leq t+w, \\ x^{n}-r_{i-t-w}, & t+w+1 \leq i \leq t+w+s .\end{cases}
$$

Then, we compute $h(y)=G \cap Q[y]$ and take

$$
m(y)=\frac{h(y)}{\lambda}, \lambda=l c(h(y)) .
$$

Hence, $m(y)$ is the minimal polynomial of $\alpha$ in $Q$. And then we can rationalize the denominator by applying the minimal polynomial.

Set $n(y)=x+y$ ( $x$ is a parameter) and divide $m(y)$ by $n(y)$ such that

$$
m(y)=q(y) n(y)+r(y), \partial(r(y))<\partial(n(y)) .
$$

Then, $\partial(r(y))=0$ and $r(y) \neq 0$, otherwise, it contradicts that $n(y)$ contains $x$. Correspondingly, equation (38) can be rewritten as

$$
m(y)=q(y) n(y)+r(x), r(x) \in Q[x] .
$$

Substitute $y=\alpha$ into the equation above, and then, $0=(x+\alpha) q(\alpha)+r(x)$, that is,

$$
\frac{1}{x+\alpha}=-\frac{q(\alpha)}{r(x)}
$$

which is the desired result of rationalizing denominators.

\section{Algorithm and Example}

According to the theorems and discussion in Section 3, we obtain an algorithm for rationalizing the denominators of fractions with the form as $1 /\left(x+c_{1} \cdot \sqrt[m]{a}+c_{2} \cdot \sqrt[n]{b}\right)$. We describe this algorithm in more detail in Figure 1.

In the following, we construct an example to show the effectiveness of the algorithm.

Example 1. Rationalizing the denominator of

$$
\frac{1}{x+\sqrt{6}+\sqrt[3]{4}}
$$

Step 1: we know $a=6$ and $b=4$, and the command "format rat" can be omitted here. Carry out the prime factorization of 6 and 4 by using the function "factorization," and we obtain

$$
6=2 \times 3,4=2 \times 2 .
$$

It is easy to see that $p_{1}=2$ is a common prime factor of 6 and 4 . In addition, 6 also contains the factor $q_{1}=3$.

Step 2: note that $m=2$ and $n=3$, and then, using the function "min_GBS," we obtain

$$
l=\operatorname{lcm}(m, n)=6 .
$$

Let

$$
\alpha_{1}=\sqrt[6]{2}, \alpha_{2}=\sqrt{3} .
$$

We construct the extension field $Q\left(\alpha_{1}, \alpha_{2}\right)$ of the rational number field $Q$. Set $\alpha=\sqrt{6}+\sqrt[3]{4}$, and then, $\alpha=f\left(\alpha_{1}, \alpha_{2}\right)$ $=\alpha_{1}^{3} \alpha_{2}+\alpha_{1}^{4}$.

Step 3: hence, $h_{1}=x_{1}^{6}-2 \in \mathrm{Q}\left[x_{1}\right]$ is a minimal polynomial of $\alpha_{1}$ on $Q$ and $h_{2}=x_{2}^{2}-3 \in Q\left(\alpha_{1}\right)\left[x_{2}\right]$ is a minimal polynomial of $\alpha_{2}$ on $Q\left(\alpha_{1}\right)$.

Set

$$
I=\left\langle x_{1}^{6}-2, x_{2}^{2}-3, y-x_{1}^{3} x_{2}-x_{1}^{4}\right\rangle,
$$

take the lexicographical ordering $y<x_{2}<x_{1}$ as the term order in $Q\left[x_{1}, x_{2}, y\right]$, and calculate the reduced Gröbner basis $G$ of ideal $I$ in the software singular using the built-in function "groebner." Then, the calculation results are as follows:

$$
G=\left\{f_{1}, f_{2}, f_{3}\right\},
$$

where 


\begin{tabular}{|c|c|}
\hline \multicolumn{2}{|r|}{ An algorithm for rationalizing denominator } \\
\hline $\begin{array}{l}\text { Input: } \\
\text { Output: }\end{array}$ & $\begin{array}{l}m, n \in N^{+}, a, b \in Q^{+}, c_{1}, c_{2} \in Q, \text { the term orders over } Q \\
\alpha, q(x), r(x) \text { such that } \frac{1}{x+\alpha}=-\frac{q(\alpha)}{r(x)}\end{array}$ \\
\hline Step 1. & $\begin{array}{l}\text { Write } a, b \text { as fractions in lowest term } a=d_{1} / e_{1}, b=d_{2} / e_{2} \text { using } \\
\text { the command "format rat". Then decompose } d_{i}, e_{i}(i=1,2) \text { into } \\
\text { power product of different prime factors respectively using the func- } \\
\text { tion "factorization", denote the common prime factors of } d_{1}, d_{2} \text { by } \\
p_{i} \text {, use } q_{j} \text { and } r_{k} \text { to denote the different prime factors in } d_{1}, e_{1} \text { and } \\
d_{2}, e_{2} \text { respectively, } i, j, k=1,2, \ldots\end{array}$ \\
\hline Step 2. & $\begin{array}{l}\text { Compute } l=\operatorname{lcm}(m, n) \text {, set } \alpha_{i}=\sqrt[l]{p_{i}}, i=1,2, \ldots, t ; \alpha_{t+j}=\sqrt[l]{q_{j}}, \\
j=1, \ldots, w \text {, and } \alpha_{t+w+k}=\sqrt[l]{r_{k}}, k=1, \ldots, s \text {. Construct the ex- } \\
\text { tension field } M=Q\left(\alpha_{1}, \alpha_{2}, \ldots, \alpha_{t+w+s}\right) \text {. Find } f\left(x_{1}, \ldots, x_{t+w+s}\right) \text {, } \\
g\left(x_{1}, \ldots, x_{t+w+s}\right) \in Q\left(x_{1}, \ldots, x_{t+w+s}\right) \text { such that } \alpha=\frac{f\left(\alpha_{1}, \ldots, \alpha_{t+w+k}\right)}{g\left(\alpha_{1}, \ldots, \alpha_{t+w+k}\right)} .\end{array}$ \\
\hline Step 3. & $\begin{array}{l}\text { Let } h_{i}=x^{l}-p_{i} \text { for } i=1,2, \ldots, t, h_{t+j}=x^{m}-q_{j} \text { for } j=1, \ldots, w \text {, and } \\
h_{t+w+k}=x^{n}-v_{k} \text { for } k=1, \ldots, s, \text { set } I=\left\langle h_{1}, \ldots, h_{t+w+k}, g \cdot y-f\right\rangle \text {, } \\
\text { calculate the reduce Gröbner basis } G \text { of } I \text { using the function "groeb- } \\
\text { ner". Then compute } h(y)=G \cap Q[y] \text {. Set } m(y)=h(y) / l c(h(y)) \text {, } \\
n(y)=x+y \text { divide } m(y) \text { by } n(y) \text { using the function "polynomialRe- } \\
\text { duce" such that } m(y)=q(y) n(y)+r(y), \quad \partial(r(y))<\partial(g(y)) \text {. Write } \\
m(y)=q(y) n(y)+r(x) \text {, and substitute } y=\alpha \text { in the equation. } \\
\alpha, q(x), r(x) \text {. }\end{array}$ \\
\hline
\end{tabular}

FIGURE 1: Denominator rationalized algorithm.

$$
\begin{aligned}
& f_{1}=y^{6}-18 y^{4}+108 y^{2}-144 y-200, \\
& f_{2}=x_{2}^{2}-3 \\
& f_{3}=7128 x_{1}+18 x_{2}^{3} y^{4}-144 x_{2}^{3} y^{3}-294 x_{2}^{3} y^{2}+4756 x_{2}^{3} y+64 x_{2}^{3}-3 x_{2} y^{5}-30 x_{2} y^{2}-14904 x_{2} y+4536 x_{2} .
\end{aligned}
$$

Therefore, $G \cap Q[y]=\left\{y^{6}-18 y^{4}-8 y^{3}+108 y^{2}-144 y\right.$ $-200\}$. Hence, the minimal polynomial of $\alpha$ in the rational number field $Q$ is $m(y)=y^{6}-18 y^{4}-8 y^{3}+108 y^{2}-$ $144 y-200$.

Next, divide $m(y)$ by $x+y$ in the software Matlab using the built-in function "polynomialReduce." Then,

$$
\begin{aligned}
& q(y)=y^{5}-x y^{4}+y^{3}\left(x^{2}-18\right)+y^{2}\left(-x^{3}+18 x-8\right)+y\left(x^{4}-18 x^{2}+8 x+108\right)+\left(-x^{5}+18 x^{3}-8 x^{2}-108 x-144\right) \\
& r(x)=x^{6}-18 x^{4}+8 x^{3}+108 x^{2}+144 x-200 .
\end{aligned}
$$

Substitute $\alpha=\sqrt{6}+\sqrt[3]{4}$ into

$$
\frac{1}{x+\alpha}=-\frac{q(\alpha)}{r(x)}
$$

$$
\begin{aligned}
\frac{1}{x+\sqrt{6}+\sqrt[3]{4}}= & \frac{x^{5}-12 x^{3}+4 x^{2}+36 x+24}{r(x)}+\frac{-x^{4}+12 x^{2}+8 x-36}{r(x)} \sqrt{6}+\frac{-x^{4}-4 x+36}{r(x)} \sqrt[3]{4} \\
& +\frac{2 x^{3}+36 x+8}{r(x)} \sqrt[3]{2}+\frac{2 x^{3}-12 x-4}{r(x)} \sqrt{6} \sqrt[3]{4}+\frac{-6 x^{2}-12}{r(x)} \sqrt[3]{2} \sqrt{6} .
\end{aligned}
$$


Remark 1. Note that $m=2$ and $n=3$, and we can use the method in Section 2 to do this example. Replace $a=6$ and $b=4$ and do all the steps as in Section 2, and we obtain

$$
\begin{aligned}
\frac{1}{x+\sqrt{6}+\sqrt[3]{4}}= & \frac{1}{x^{6}-18 x^{4}+8 x^{3}+108 x^{2}+144 x-200}\left(x^{5}-12 x^{3}+4 x^{2}+36 x+24+\left(-x^{4}+12 x^{2}+8 x-36\right)\right. \\
& \cdot \sqrt{6}+\left(-x^{4}-4 x+36\right) \cdot \sqrt[3]{4}+\left(2 x^{3}+36 x+8\right) \cdot \sqrt[3]{2} \\
& \left.+\left(2 x^{3}-12 x-4\right) \cdot \sqrt{6} \cdot \sqrt[3]{4}+\left(-6 x^{2}-12\right) \cdot \sqrt{6} \cdot \sqrt[3]{2}\right)
\end{aligned}
$$

Through the two examples above, it is easy to find that the results obtained by both methods are the same, which proves that the algorithm we designed is correct and the application scope is wider.

Remark 2. Using the method in Section 2 or the method of undetermined coefficients, the problem of denominator rationalization of fractions with the form as $1 /\left(x+c_{1} \cdot \sqrt[m]{a}+\right.$ $\left.c_{2} \cdot \sqrt[n]{b}\right)$ may not be solved or can be solved but will take a long time. However, from Example 1, it is straightforward that this problem can be solved efficiently and simply according to the three steps of Denominator rationalized algorithm by using the software Matlab and Singular.

\section{Conclusion}

In this paper, using theory of Gröbner bases, we have achieved in rationalizing denominators for two types of fractions, especially rationalizing the denominator of the fraction with the form as

$$
\frac{1}{x+c_{1} \cdot \sqrt[m]{a}+c_{2} \cdot \sqrt[n]{b}}
$$

where $m, n \in N^{+}, m, n \geq 2, a, b, c_{1}, c_{2} \in Q, a, b>0$. We have presented some interesting results and an executable algorithm on rationalizing the denominators for this type fractions. Furthermore, we have established an example to illustrate the effectiveness of the algorithm.

The method that we proposed on rationalizing denominators can be realized in computer system such as Maple and Singular, which makes the related computation more quick. Furthermore, it can improve the performance of the related algorithms. For example, we find that, for some new swarm intelligence algorithms proposed in recent years, such as monarch butterfly optimization (MBO) [27], earthworm optimization algorithm (EWA) [28], elephant herding optimization (EHO) [29], etc. the fraction of the form $1 /\left(x+c_{1} \cdot \sqrt[m]{a}+c_{2} \cdot \sqrt[n]{b}\right)$ occurs during the implementation of these algorithms. The denominator rationalized algorithm we proposed can rationalize the denominator of this type fraction, which can improve the accuracy of these algorithms on calculation.

Future work will investigate in rationalizing denominators of more general forms of radical fraction. The type of fractions discussed in the paper is unnested radical expressions, and the problem of the nested radical fractions is not involved here, which is also what we will do.

\section{Data Availability}

Data used to support the findings of this study are included within the article.

\section{Conflicts of Interest}

The authors declare that there are no conflicts of interest regarding the publication of this paper.

\section{Acknowledgments}

This research was supported by the National Natural Science Foundation of China (11871207 and 11971161).

\section{References}

[1] A. Berele and S. Catoiu, "Rationalizing denominators," Mathematics Magazine, vol. 88, no. 2, pp. 121-136, 2015.

[2] Schuette and H. Paul, "Rationalizing the denominator: why bother?" Mathematics and Computer Education, vol. 32, no. 1, pp. 19-29, 1998.

[3] D. C. De, "Learn to realize the "solution" engendered by skills: ideals from a "denominator rationalization" teaching short video," The World of Junior School Student: China Version, vol. 12, pp. 44-88, 2021.

[4] E. H. Neville, "Partial fractions rationalizing the denominator," The Mathematical Gazette, vol. 42, no. 342, pp. 261-266, 1958.

[5] H. B. Li, "A method for denominator rationalzation of higher radical fraction," Middle-school Mathematics: China version, vol. 6, pp. 23-24, 1982.

[6] L. J. Paradiso, "Discussions: rationalizing factors and the method of undetermined coefficients," The American Mathematical Monthly, vol. 36, no. 2, pp. 87-89, 1929.

[7] B. F. Caviness and R. J. Fateman, "Simplification of radical expressions," in Proceedings of the 1976 ACM Symposium on Proceedings of the third ACM Symposium on Symbolic and Algebraic Computation, pp. 329-338, Association for Computing Machinery, New York, NY, United States, August 1976.

[8] J. Zhou, "One way to rationalize the denominator for $1 /(\mathrm{g}(\mathrm{u}))$, the middle school teaching," Mathematics version, vol. 3, pp. 38-40, 1986. 
[9] H. C. Ma, "On the discussion of denominator's rationalization of irrational expressions," Journal of Yangzhou Teachers college(Natural Science): China version, pp. 10-13, 1989.

[10] J. Liu, "Use the determinant to rationalize the denominator of an algebraic expression," Journal of Mathematics: China version, vol. 11, pp. 41-42, 2000.

[11] M. M. Tang, "How to rationalize the deominators of a kind of algebraic expressions by multinomials," Journal of Zhuzhou Teachers College: China Version, vol. 5, pp. 31-33, 2002.

[12] B. Buchberger, "A criterion for detecting unnecessary reductions in the construction of Gröbner bases," Symbolic and Algebraic Computation, vol. 72, pp. 3-21, 1979.

[13] B. Buchberger, An Algorithm for Finding a Basis for the Redidue Class Ring of a Zero Dimensiomal Polynomial, phD thesis, Universität Innsbruck, Institut für Mathematik, 1965.

[14] W. Adams and P. Loustaunau, An Introduction to Gröbner Bases, American Mathematical Society, New York, 1994.

[15] T. Becker and V. Weispfenning, Gröbner Bases-A Computational Approach to Commutative Algebra, Vol. 141, GTM Springer, , New York, 1993.

[16] B. Buchberger and F. Winkler, Gröbner Bases and Application. London Mathematical Society Lecture Note Series, Vol. 251, Cambridge University Press, , Cambridge, 1998.

[17] D. Li, J. Liu, and L. Zheng, "On the equivalence of multivariate polynomial matrices," Multidimensional Systems and Signal Processing, vol. 28, no. 1, pp. 225-235, 2017.

[18] D. Li, J. Liu, and L. Zheng, "A zero-dimensional valuation ring is 1-Gröbner," Journal of Algebra, vol. 484, pp. 334-343, 2017.

[19] Y. Liu and H. Su, "Some necessary and sufficient conditions for containment of second-order multi-agent systems with sampled position data," Neurocomputing, vol. 378, pp. 228-237, 2020.

[20] Y. Liu and H. Su, "Containment control of second-order multi-agent systems via intermittent sampled position data communication," Applied Mathematics and Computation, vol. 362, p. 124522, 2019.

[21] C. Xu, H. Xu, H. Su, and C. Liu, "Disturbance-observer based consensus of linear multi-agent systems with exogenous disturbance under intermittent communication," Neurocomputing, vol. 404, pp. 26-33, 2020.

[22] C. $\mathrm{Xu}, \mathrm{B}$. Li, and L. Yang, "Semi-global containment of discrete-time high-order multi-agent systems with input saturation via intermittent control," IET Control Theory and Applications, vol. 14, no. 16, pp. 2303-2309, 2020.

[23] D. A. Cox, J. Little, and D. O'Shea, Ideals, Varieties, and Algorithms: An Introduction to Computational Algebraic Geometry and Commutative Algebra, Springer, New York, 2007.

[24] S. Lang, "Algebraic number theory," Graduate Texts in Mathematics, Springer, New York, Second Edition, 1994.

[25] D. Eisenbud, Commutative Algebra with a View towards Algebraic Geometry, Springer-Verlag, New York, 1995.

[26] D. Wang, B. Xia, and Z. Li, Computer Algebra, pp. 181-183, Tsinghua University Press, Beijing, 2007.

[27] Y. H. Feng, S. Deb, G. G. Wang, and H. A. Alavi, "Monarch butterfly optimization: a comprehensive review," Expert Systems with Applications, vol. 168, p. 2021.

[28] S. K. Kanna, Rajesh, K. Sivakumar, and N. Lingaraj, "Development of deer hunting linked earthworm optimization algorithm for solving large scale traveling salesman problem," Knowledge-Based Systems, vol. 227, 2021.

[29] J. Li, H. Lei, H. A. Alavi, and G. G. Wang, "Elephant herding optimization: variants, hybrids, and applications," Mathematics, vol. 8, no. 9, p. 2020. 